\title{
Identification of functional modules using network topology and high-throughput data Igor Ulitsky and Ron Shamir*
}

\author{
Address: School of Computer Science, Tel Aviv University, Tel Aviv 69978, Israel \\ Email: Igor Ulitsky - ulitskyi@tau.ac.il; Ron Shamir* - rshamir@tau.ac.il \\ * Corresponding author
}

Published: 26 January 2007

BMC Systems Biology 2007, I:8 doi: 10.I 186/I752-0509-I-8

This article is available from: http://www.biomedcentral.com/I752-0509///8

(C) 2007 Ulitsky and Shamir; licensee BioMed Central Ltd.

This is an Open Access article distributed under the terms of the Creative Commons Attribution License (http://creativecommons.org/licenses/by/2.0), which permits unrestricted use, distribution, and reproduction in any medium, provided the original work is properly cited.
Received: 18 September 2006

Accepted: 26 January 2007

\begin{abstract}
Background: With the advent of systems biology, biological knowledge is often represented today by networks. These include regulatory and metabolic networks, protein-protein interaction networks, and many others. At the same time, high-throughput genomics and proteomics techniques generate very large data sets, which require sophisticated computational analysis. Usually, separate and different analysis methodologies are applied to each of the two data types. An integrated investigation of network and highthroughput information together can improve the quality of the analysis by accounting simultaneously for topological network properties alongside intrinsic features of the high-throughput data.

Results: We describe a novel algorithmic framework for this challenge. We first transform the highthroughput data into similarity values, (e.g., by computing pairwise similarity of gene expression patterns from microarray data). Then, given a network of genes or proteins and similarity values between some of them, we seek connected sub-networks (or modules) that manifest high similarity. We develop algorithms for this problem and evaluate their performance on the osmotic shock response network in S. cerevisiae and on the human cell cycle network. We demonstrate that focused, biologically meaningful and relevant functional modules are obtained. In comparison with extant algorithms, our approach has higher sensitivity and higher specificity.
\end{abstract}

Conclusion: We have demonstrated that our method can accurately identify functional modules. Hence, it carries the promise to be highly useful in analysis of high throughput data.

\section{Background}

The accumulation of large-scale interaction data on multiple organisms, such as protein-protein and protein-DNA interactions, requires novel computational techniques that will be able to analyze these data together with information collected through other means. Such methods should enable thorough dissection of the data, whose dimensions have already extended far beyond the scope that is amenable to traditional analysis and manual interpretation. An important class of such biological information can be represented in the form of similarity relations. Quantitative molecular data, such as mRNA expression profiles, are often analyzed in this context through clustering algorithms. Similarity between genes can also be defined on other levels, such as function [1] or transcription factor binding patterns [2].

Although many fruitful algorithmic approaches have been developed for dissection of network and similarity data separately, methods analyzing together both information sources hold much promise. Several works have established the interconnection between expression profile similarity and protein interactions $[3,4]$. To exploit this interconnection, pairwise gene expression similarities 
have been used together with other data sources for predicting pairwise protein interactions (e.g., [5]). Topological properties of interaction networks induced by genes active in different conditions were studied [6-9]. Several software tools allow the visual inspection of the clustering results in a network context [10]. However, ignoring the network information in the clustering process and using the rich and constantly growing network information solely for cluster evaluation seems suboptimal, as the network information can improve the cluster identification process. The prevalence of modularity in molecular cell biology has been widely recognized in the last decade. By functional module one typically means a group of cellular components and their interactions that can be attributed a specific biological function [11]. Several approaches sought modules by jointly analyzing network information with gene expression data. Initial works [12,13] proposed measures for scoring expression activity in metabolic pathways (e.g. KEGG database [14]) and complexes [15]. Vert and Kanehisa [16] used kernel methods to identify expression patterns that characterize gene sets matching pathways in a given network.

The Co-clustering methodology [17] uses a distance function that combines similarity of gene expression profiles with network topology. The network distance between two nodes is an edge-weighted version of their topological distance in the network. The expression distance is one minus the Pearson correlation between the expression patterns. The two distances are combined into a similarity score, and standard hierarchical algorithms [18] are used for clustering. While generally successful, this approach sometimes produces clusters corresponding to highly disconnected subnetworks, since the network is only used as one of the sources of distance information, without requiring connectivity.

Ideker et al. [19] introduced a successful algorithm for identification of active subnetworks, i.e., connected regions of the network that show significant changes in expression over a particular subset of the conditions. Unfortunately, this method can be used only when one has an activity pvalue for every measurement, a situation which is rather uncommon. In addition, the method cannot handle pairwise gene similarity input. The same methodology was recently used in [20], utilizing shortest-path algorithms for module finding. Segal et al. [21] provided another interesting formulation of the integration problem, in which a module is expected to contain a significant portion of the possible interactions. A probabilistic graphical model was used to extract a prespecified number of modules from gene expression measurements combined with a protein interaction dataset.
In this study we seek functional modules by identifying connected subnetworks in the interaction data that exhibit high average internal similarity. We call such a module a Jointly Active Connected Subnetwork (JACS). By imposing network topology constraints on clusters of expression data, the biological interpretation of the clusters becomes easier, and, as we shall see, one can detect weaker signals that were indistinguishable by extant methods.

We develop a novel computational method for efficient detection and analysis of JACSs, implemented in a program called MATISSE (Module Analysis via Topology of Interactions and Similarity SEts). The proposed methodology has a statistical basis, which allows confidence estimation of the results. The algorithm assumes no prior knowledge on the number of JACSs, and allows imposing constraints on their size. We do not require precalculation of the statistical significance of expression values. The methodology is general enough to suit any type of network data overlaid with pairwise similarities.

Our algorithm detects JACSs by identifying heavy subgraphs in an edge-weighted similarity graph while maintaining connectivity in the interaction network. By transforming edge weights to attain probabilistic meaning, we are actually seeking subnetworks of maximum likelihood. We show that this problem is computationally hard, devise several heuristic methods and analyze their practical performance.

When using gene expression similarity, analysis of known pathways in yeast has shown that only a fraction of the genes in a pathway are usually coherently regulated at the transcription level (and thus highly similar) [22]. Our method allows assignment of different priors to different genes, reflecting their probability to be regulated at the transcription level. We believe this is the first study to allow such flexibility. In addition, the goal of our approach is to detect non-overlapping JACSs rather than to partition all the genes into clusters.

We first evaluate the performance of our algorithm on synthetic data with planted modules, and verify its ability to recover planted modules with high accuracy. Then, we analyze two real systems for which large datasets are available: the osmotic shock response of $S$. cerevisiae, and the cell cycle in human HeLa cells. For $S$. cerevisiae, we compiled and carefully annotated from diverse sources a protein-protein and protein-DNA interaction network consisting of 6,230 nodes and 89,327 interactions. The performance of MATISSE is shown to exceed that of extant analysis schemes in terms of the ability to retrieve biologically relevant groups, as analyzed by four different anno- 
tation datasets. We identify specific subnetworks relevant to different processes that are known to be activated and repressed by the MAPK cascades following osmotic shock, such as ergosterol biosynthesis and pheromone response. In addition, we identify novel pathways, such as pyridoxine metabolism, as differentially expressed during osmotic shock. Detailed analysis shows that some of the involved processes can not be detected based on the expression data alone. The human network contains 9,135 nodes and 25,086 protein-protein interactions collected from several sources, including recently published studies $[23,24]$. Our analysis identifies subnetworks active in specific phases of the human cell cycle. These results underly the ability of our approach to provide novel, previously undetected biological insights. The inspection of "hubs" in the subnetworks delineated by MATISSE reveals key regulators of the cell cycle.

\section{Results and discussion}

\section{A framework for detection of jointly active subnetworks}

Let us first state our problem abstractly. We are given an undirected constraint graph $G^{C}=(V, E)$, a subset $V_{\text {sim }} \subseteq V$ and a symmetric matrix $S$ where $S_{i j}$ is the similarity between $v_{i}, v_{j} \in V_{\text {sim }}$. The goal is to find disjoint subsets $U_{1}$, $U_{2}, \ldots, U_{m} \subseteq V$ called JACSs, so that each JACS induces a connected subgraph in $G^{C}$ and contains elements that share high similarity values. We call the nodes in $V_{\text {sim }}$ front nodes and nodes in $V \backslash V_{\text {sim }}$ back nodes.

In the biological context, $V$ represents genes or gene products (we shall use the term 'gene' for brevity), and $E$ represents interactions between them. These can be known protein-protein or protein-DNA interactions or alterna- tively can originate from a known regulatory network where arc orientations are ignored. $S_{i j}$ measures the similarity between genes $i$ and $j$, e.g., the Pearson correlation between their gene expression patterns. The set $V_{\text {sim }}$ may be smaller than $V$ as some of the genes may be absent from the array, and others may show insignificant expression patterns across the tested conditions and thus excluded. Hence, a JACS aims to capture a set of genes that have highly similar behavior, and are also topologically connected, and thus may share a common function, e.g., belong to a single complex or pathway. As elaborated in Methods, we formulate the problem of JACS identification as a hypothesis testing question. In this approach statistically significant JACSs correspond to heavy subnetworks in a similarity graph, with nodes inducing a connected subgraph in $G^{C}$ (Figure 1). The probabilistic model we propose also accommodates the use of genespecific priors, reflecting our confidence that they are transcriptionally regulated in the studied conditions.

As exact optimization is intractable, we designed and tested several heuristics for solving the problem (see Methods). The version that performed best on real biological data had the following three phases: (1) detection of relatively small, high-scoring gene sets, or seeds; for each node, the set consisting of it along with the neighboring nodes that are connected to it via positive-weighted edges was a candidate seed; (2) seed improvement, and (3) significance-based filtering (see Methods for full details). This version, which we call MATISSE, was used in subsequent analysis.

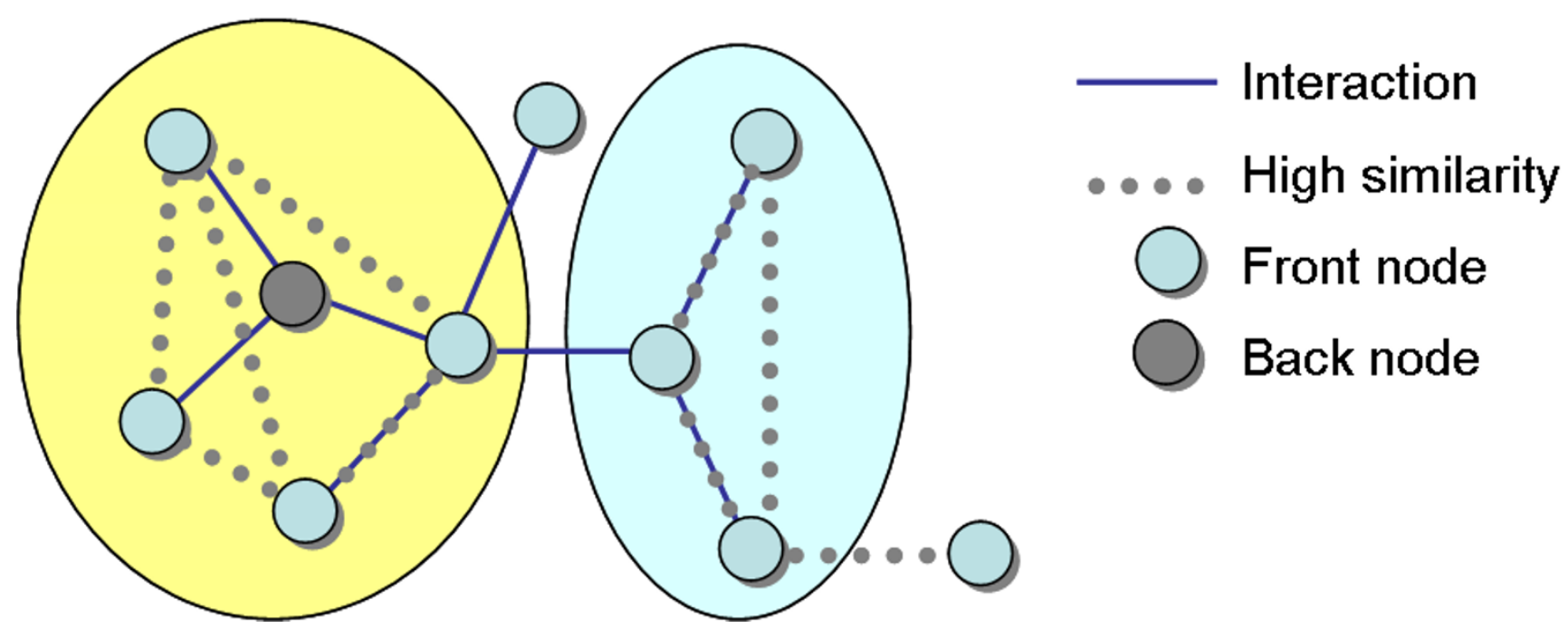

Figure I

Toy input example. A toy example of an input problem with two distinct JACSs and with front and back nodes. Both JACSs (circled) are connected in the interaction network and heavy in the similarity graph. Note that the four front nodes in the left JACS form a connected subgraph only after the addition of the back node. 


\section{Analysis of performance using simulated similarity values} In order to evaluate the ability of our method to detect subnetworks of high pairwise similarity, we first tested its performance on simulated similarity data. The simulation used a connected subnetwork of 2,000 nodes from the $S$. cerevisiae interaction network (described below) as the constraint graph. The similarity data were generated by "planting" a collection of JACSs with several defining parameters in the network, using two similarity value distributions, where members of the same JACS tend to have higher similarity, as described in Methods.

In order to test the effect of each parameter on the performance of the different module finding algorithms, we carried out simulations in which one parameter was varied while keeping the rest at their default values. We also tested simple clustering of the similarity data with the $K$ means algorithm and with the Co-clustering approach of Hanisch et al. [17], which proposes a distance measure based on topology and expression. Since the latter method does not readily provide clusters, we used that measure with a $K$-means-like algorithm (with $K=15$, and moving genes between clusters based on average distance). Other methods (e.g., [21]) were not readily available for comparison.

We evaluated the ability of the methods to recover the planted components using Jaccard coefficient. The coefficient ranges between 0 and 1 with 1 indicating perfect recovery (see Methods). The results are presented in Figure 2. MATISSE is able to retrieve the planted components with good precision when there is a plausible separation between the two similarity value distributions (above 1.3 standard deviations) and the fraction of the front nodes exceeds 0.8 . The performance of MATISSE exceeds that of other methods for most of the parameter range.

\section{Response to osmotic stress in S. cerevisiae}

We generated a comprehensive $S$. cerevisiae protein-protein and protein-DNA interaction network by combining information from the interaction databases SGD, BioGRID and BIND and recent high-throughput studies (e.g., [25], see our website for a complete list). This resulted in a network containing 6,230 nodes and 89,327 interactions. We also used 133 expression profiles of $S$. cerevisiae under different perturbations and different environmental conditions focused on the osmotic stress response [26]. The 2,000 genes whose patterns exhibit the highest variation in the data were designated as front nodes. We used Pearson correlation for scoring similarities between expression patterns. The parameters of the probabilistic model were assigned as described in Methods. Maps of the subnetworks produced by MATISSE are provided on our website and in the supplement [see Additional file 1].
Comparison of the modules produced by each method

We compared the performance of MATISSE to Co-clustering and to clustering based solely on the gene expression data. We used the CLICK algorithm [27] for clustering, as it was shown to outperform several extant gene expression clustering algorithms, and since it can determine the number of clusters and also leave some vertices unclustered. The Ideker et al. method [19] could not be tested in this setting, since measurement p-values could not be computed.

Table 1 compares the properties of the modules produced by every method. Expression homogeneity is calculated as the average Pearson correlation between genes within the same module. The edge density of a subgraph is the number of edges it contains as a fraction of all its node pairs. The clustering coefficient of a node is the fraction of its neighbor pairs that are connected in the network [28]. The clustering coefficient of a module is the average coefficient in the subgraph induced by the module. In the "Random connected" and "Random" solutions, modules were randomly sampled gene groups with and without the requirement for network connectivity, respectively. The sizes of the random groups were matched to the sizes obtained by MATISSE.

\section{Expression homogeneity}

As expected, the most homogeneous clusters in terms of expression similarity are obtained by CLICK, which optimized this type of similarity. The homogeneity of the MATISSE JACSs is higher than that of co-clusters. As previously reported [3], the expression homogeneity of a random connected set is higher than that of a random arbitrary set (average coherence of 0.063 for the random connected solution, vs. 0.033 for random arbitrary solution).

\section{Topological descriptors}

MATISSE is designed to produce connected subnetworks. The significance of this criterion is evident from the comparison to the other algorithms. In contrast to MATISSE, both CLICK and Co-clustering produce modules that are highly disconnected (averaging 80-90 components per module). Interestingly, the subnetworks produced by MATISSE are not denser than random connected components in the network. This observation can be explained by the fact that the network contains several dense complexes that do not participate in the solutions, as their components are not homogeneously expressed under the examined conditions.

\section{Functional enrichment}

In order to compare the functional relevance of the modules found by the different methods we used four annotation databases: (a) GO "biological process" ontology (level 7; 474 categories) [29]; (b) GO complexes annotation (subterms of "protein complex" term, 213 com- 
(a)

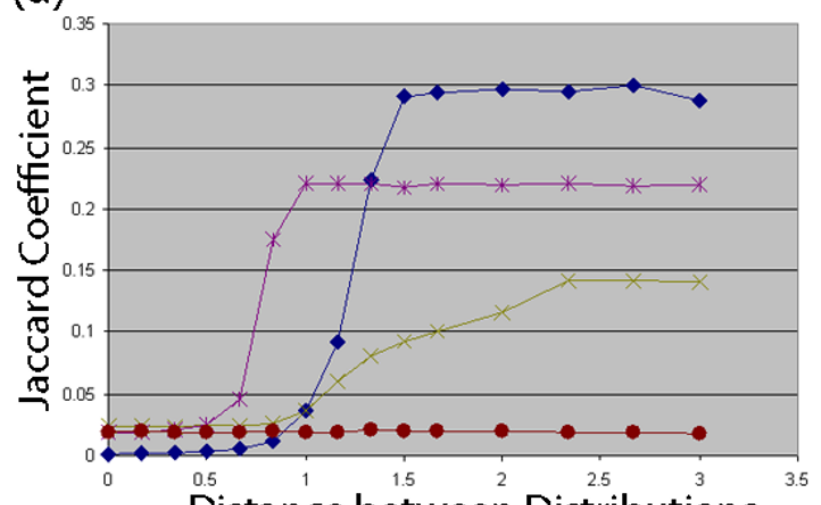

(c)

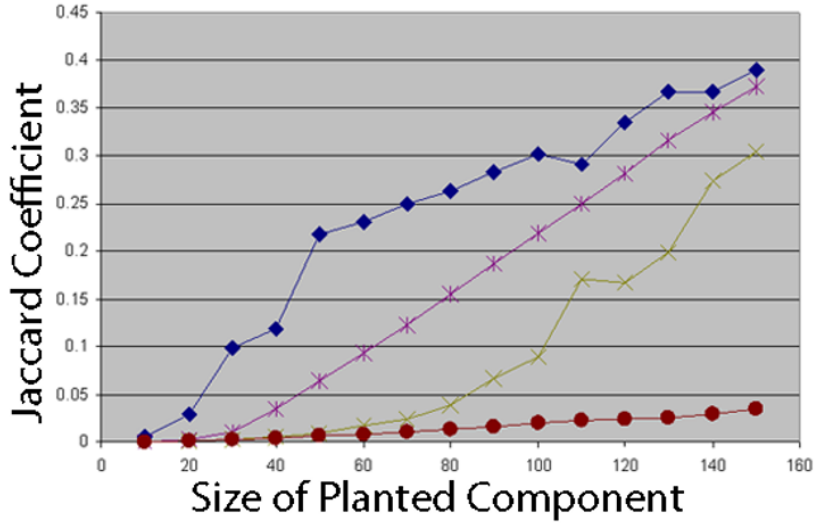

(b)
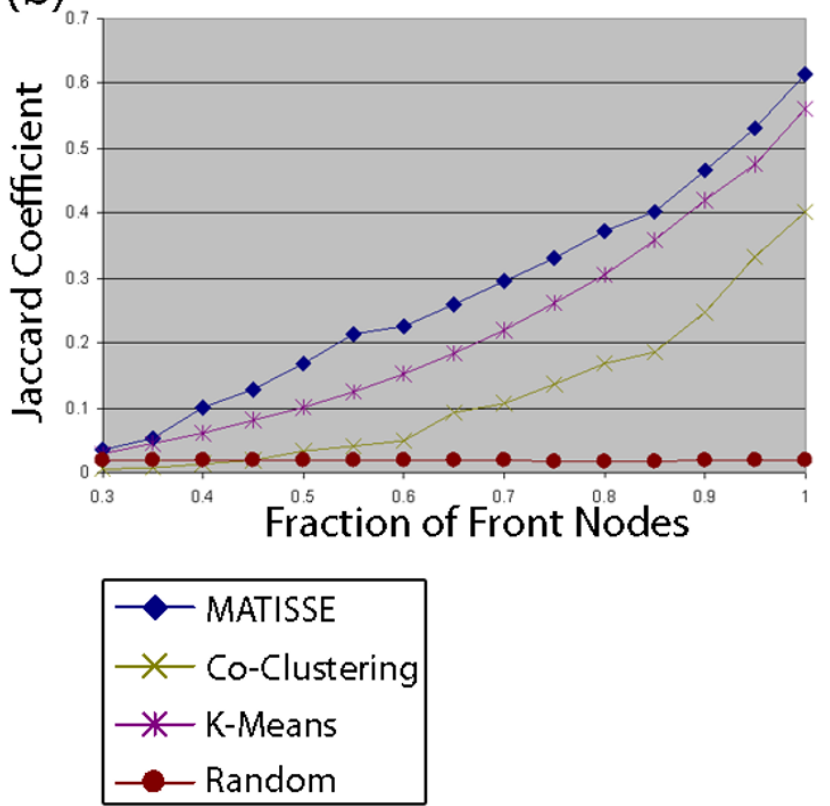

Figure 2

Performance of different module finding procedures on simulated data. Co-clustering: clustering based on the distance metric of [17]. K-Means: clustering of the similarity data. Random: random sampling of connected subnetworks matched in size and number to the planted components. The quality of solutions produced by the different procedures is evaluated by the Jaccard coefficient, (a) Performance as a function of the distance between the means of the mates and the non-mates distributions $\left(\mu_{m}\right)$. (b) Performance as a function of the fraction of front nodes $\left(p_{f}\right)$. (c) Performance as a function of planted component size $(k)$.

Table I: Performance of the different module finding algorithms on the S. cerevisiae osmotic shock data

\begin{tabular}{lccccccc}
\hline Solution & No. of modules & Total nodes & Average size & $\begin{array}{c}\text { Expression } \\
\text { homogeneity }\end{array}$ & $\begin{array}{c}\text { Clustering coefficient } \\
\text { Edge density }\end{array}$ & $\begin{array}{c}\text { No. of connected } \\
\text { components }\end{array}$ \\
\hline MATISSE & 20 & 2107 & 105.35 & 0.361 & 0.073 & 0.035 & 1.00 \\
Co-clustering & 19 & 1991 & 104.79 & 0.354 & 0.035 & 0.010 & 89.67 \\
CLICK & 20 & 1988 & 99.40 & 0.438 & 0.030 & 0.011 & 77.61 \\
Random connected & 20 & 2107 & 105.35 & 0.063 & 0.050 & 0.036 & 1.00 \\
Random & 20 & 2105 & 105.35 & 0.033 & 0.004 & 0.003 & 89.78 \\
\end{tabular}

Numbers in columns $4-8$ are averages over all the modules in each solution. 
plexes); (c) MIPS deletion phenotype annotations [30] (181 phenotypes); (d) KEGG molecular pathways (310 pathways) [14]. A relatively wide selection of annotations was used to encompass diverse biological functions. Note that the GO "molecular function" categories are not relevant here, as the identified sets of genes are not expected to have similar molecular mechanisms.

For each annotation and for each group of genes produced by every method, the hypergeometric p-value was computed (without correcting for multiple testing, see below). We analyzed the percentage of the modules (Figure 3a) and of the categories (Figure $3 \mathrm{~b}$ ) enriched with p-value $\leq$ $10^{-3}$ in each solution. MATISSE exhibits high performance in functional terms and in most cases the produced JACSs show higher enrichment than expression clusters and coclusters. Co-clustering and CLICK perform slightly better than MATISSE in covering KEGG categories. This is probably due to the overrepresentation of metabolic pathways in KEGG. Metabolic pathways are generally poor in direct protein-protein and protein-DNA interactions, and thus less likely to be recognized by MATISSE, which relies also on direct interactions, than by a clustering algorithm based on expression alone.

As an additional comparison between MATISSE and Coclustering, we compared the p-values obtained by each solution on each GO biological process (level 7) class attaining enrichment of $p \leq 0.01$ in at least one of the solutions. The MATISSE modules gave better significance to 238 functions, while only 116 functions had higher significance in the Co-clustering solution.

In order to check the added value of incorporating network constraints over using only expression profiles, we compared the results to clustering of the expression pro- files with CLICK. In the same pairwise comparison, 223 MATISSE functions exhibited a higher enrichment, compared to 146 in CLICK. Several relevant functions, such as pyridoxine metabolism, cellular response to phosphate starvation, protein ubiquitination and post-Golgi transport, were enriched with $p<10^{-5}$ in MATISSE, but were not significantly enriched in any CLICK cluster. When seeking functions enriched by the other clustering methods, the only function enriched was "NAD biosynthesis" $\left(p<10^{-5}\right)$ discovered by CLICK. The six genes in our dataset that are annotated with this category do not contain any interactions between them and the average length of the shortest path between them is 7 .

\section{Functional subnetworks identified by MATISSE}

In the previous analysis we did not correct for multiple testing since our goal was the comparison of the different methods. To address the multiple testing problem, we performed a GO functional enrichment analysis using the TANGO algorithm [31]. The algorithm considers all levels of the GO hierarchy and provides p-values corrected for multiple testing and for category dependency using resampling (see Methods).

21 distinct functional terms were found to be enriched ( $p$ $<0.05)$ in 14 distinct modules. The complete list of the enriched functions and their respective JACSs is shown in Table 2. Interactive maps of these JACSs can be found at our website along with the corresponding expression data. Note that JACSs were artificially limited to contain no more than 120 nodes in order to provide a better separation between pathways with slightly similar expression patterns. Nevertheless, it appears that this bound does not cause substantial fragmentation of the true clusters, as almost all the JACSs were enriched with distinct functions. Reassuringly, most of the enriched functions are highly

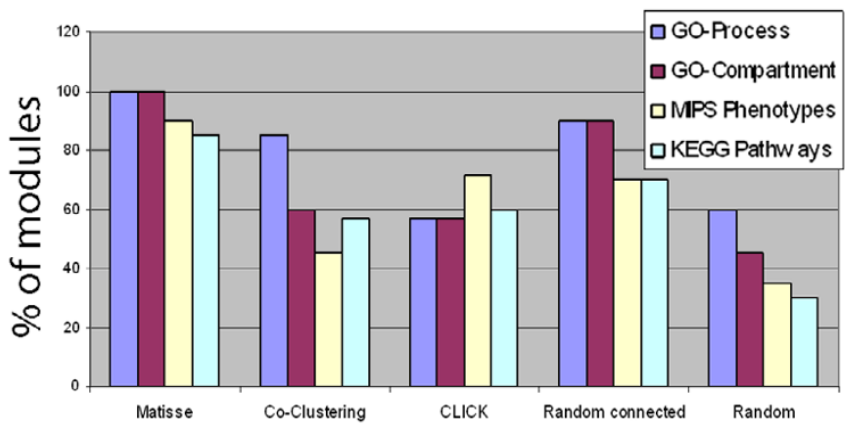

(a)

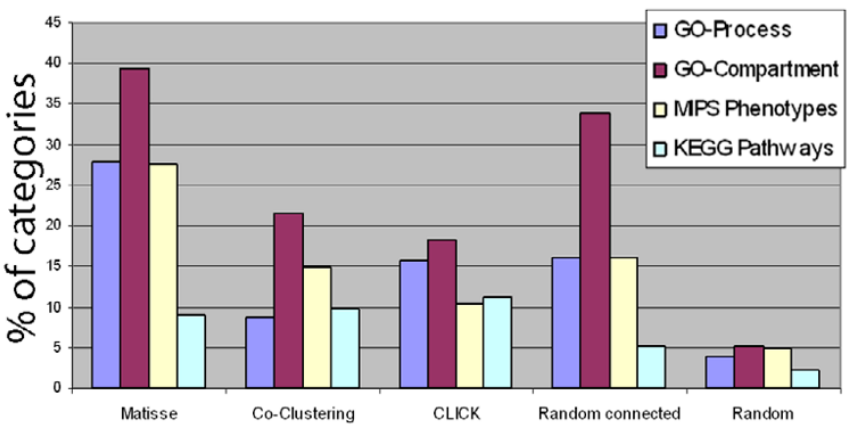

(b)

Figure 3

Performance of different module finding algorithmson S. cerevisiae osmotic shock data. (a) The fraction of the modules for which at least one category was enriched, (b) The fraction of the categories enriched in at least one module. Enrichment was defined as attaining hypergeometric $\mathrm{p}$-value $\leq 10^{-3}$. Annotation sets: GO-Process: Level 7 of the GO "biological process" ontology; GO-Complex: subterms of "protein complex" term, GO:0043234; MIPS Phenotypes: MIPS deletion phenotype annotations; KEGG Pathways: KEGG molecular pathway participation. 
relevant to the conditions and the perturbations in the data [32]. These include stress responses, such as repression of the translational machinery (JACSs 1-3) as well as general stress response genes (JACS 11 and 17). In addition, a specific subnetwork relevant to the activation of the pheromone response pathway following osmotic shock in hog1 strain [32] was identified (JACS 5). Indeed, since the HOG pathway shares protein kinases and phosphatases with other MAPK pathways, it was demonstrated that perturbations in Pbs2 or Hog1 lead to osmostressinduced stimulation of the pheromone response pathway [33].

JACS 7 contains seven genes from the yeast membrane ergosterol biosynthesis pathway which is strongly repressed following osmotic shock in the WT strain but not in hog1 strains. Lower levels of ergosterol make the membrane more compact and less flexible and hence lead to diminished transmembrane flux of glycerol, which is important for recovery from both hyper-osmotic and hypo-osmotic shock [32].
JACS 16 contains 19 genes members of the proteosome complex. 9 of these are back nodes, underlying the ability of MATISSE to use the network for linking co-activated genes with biologically relevant partners. Inspection of the expression data reveals a slight induction of the proteolysis genes following osmotic shock. This subtle response is missed when clustering solely the expression data, as no more than seven proteolysis genes are clustered together in the CLICK solution. Ubiquitin-dependent proteolytic mechanisms were linked to osmotic responses before [32], and our findings support this hypothesis.

Figure 4 shows JACSs 5 and 16 . These subnetworks demonstrate the use of different interaction types by MATISSE: JACS 5 is dominated by protein-DNA interactions, involving the transcription factors (TFs) Tec1, Kss1 and Dig1; JACS 16 is dominated by the protein interactions within the proteosome and the mitochondrial ribosome complexes. This subnetwork contains multiple back nodes linking front nodes. In fact, Table 2 shows that some JACSs make extensive use of nodes with no similarity data.

Table 2: Functionally enriched modules found in the yeast osmotic shock data

\begin{tabular}{|c|c|c|c|c|c|c|}
\hline JACS & Size & Front & Enriched GO terms & $\mathrm{p}$-value & TFs & $\mathrm{p}$-value \\
\hline \multirow[t]{6}{*}{1} & 120 & 119 & processing of $20 S$ pre-rRNA & $<0.001$ & Fhll & $4.82 \cdot 10^{-16}$ \\
\hline & & & rRNA processing & $<0.001$ & Rapl & $2.89 \cdot 10^{-11}$ \\
\hline & & & $35 \mathrm{~S}$ primary transcript processing & $<0.001$ & Sfpl & $2.98 \cdot 10^{-8}$ \\
\hline & & & ribosomal large subunit assembly and maintenance & 0.019 & & \\
\hline & & & rRNA modification & $<0.001$ & & \\
\hline & & & ribosome biogenesis & 0.029 & & \\
\hline 2 & 120 & 118 & translational elongation & $<0.001$ & Fhll & $1.03 \cdot 10^{-5}$ \\
\hline \multirow[t]{5}{*}{3} & 120 & 118 & processing of $20 \mathrm{~S}$ pre-rRNA & $<0.001$ & & \\
\hline & & & rRNA processing & 0.030 & & \\
\hline & & & $35 \mathrm{~S}$ primary transcript processing & 0.011 & & \\
\hline & & & ribosomal large subunit assembly and maintenance & 0.019 & & \\
\hline & & & ribosomal large subunit biogenesis & $<0.001$ & & \\
\hline \multirow[t]{2}{*}{5} & 120 & 112 & signal transduction during filamentous growth & 0.010 & Stel 2 & $5.41 \cdot 10^{-13}$ \\
\hline & & & conjugation with cellular fusion & $<0.001$ & Digl & $5.41 \cdot 10^{-13}$ \\
\hline \multirow[t]{2}{*}{6} & 120 & 99 & transcription from RNA polymerase III promoter & $<0.001$ & & \\
\hline & & & transcription from RNA polymerase I promoter & 0.006 & & \\
\hline \multirow[t]{2}{*}{7} & 120 & 107 & ergosterol biosynthesis & $<0.001$ & & \\
\hline & & & hexose transport & 0.019 & & \\
\hline 8 & 114 & 85 & chromatin remodeling & 0.050 & & \\
\hline \multirow[t]{2}{*}{11} & 120 & 114 & pseudohyphal growth & 0.010 & Msn2 & $3.17 \cdot 10^{-4}$ \\
\hline & & & response to stress & $<0.001$ & Msn4 & $1.82 \cdot 10^{-12}$ \\
\hline 14 & 120 & 102 & ubiquitin-dependent protein catabolism & 0.047 & & \\
\hline 15 & 120 & 96 & nuclear mRNA splicing, via spliceosome & $<0.001$ & & \\
\hline 16 & 89 & 61 & ubiquitin-dependent protein catabolism & $<0.001$ & Rpn4 & $6.44 \cdot 10^{-6}$ \\
\hline \multirow[t]{2}{*}{17} & 120 & 109 & response to stress & $<0.001$ & Msn4 & $1.74 \cdot 10^{-3}$ \\
\hline & & & mitochondrial electron transport & $<0.001$ & & \\
\hline 18 & 87 & 59 & nuclear mRNA splicing, via spliceosome & 0.012 & & \\
\hline 20 & 46 & 35 & pyridoxine metabolism & 0.045 & & \\
\hline
\end{tabular}

The GO p-value was adjusted for multiple testing using the TANGO algorithm (see Methods). Enriched TF binding site motifs were detected using the PRIMA algorithm [35]. TF $p$-values were Bonferroni corrected for multiple testing. 
For several pathways, such as pyridoxine biosynthesis, intracellular transport and chromatin-related complexes (mainly SAGA, Cdc73, COMPASS and RSC) that were linked by MATISSE to osmotic shock in S. cerevisiae, this linking is novel. Pyridoxine was recently linked to osmotic shock response in A. thaliana [34]. These findings underlie the ability of MATISSE to produce testable hypotheses and novel insights.

\section{Promoter analysis}

Based on the assumption that genes that exhibit similar expression pattern over multiple conditions are likely to be co-regulated and to share common cis-regulatory elements in their promoters, we searched for over-representation of known transcription factor binding site motifs in the promoters of the genes in each JACS. When using the PRIMA motif finding tool [35], six subnetworks showed significant enrichment $\left(p<10^{-5}\right)$ for at least one TF (Table 2 ). All the TFs corresponded to known regulators of the processes enriched in the subnetworks. For example, JACS 5 , enriched for pheromone response pathway genes, was enriched with putative targets of Dig1 and Ste12, known regulators of these pathways [36]. Subnetwork 11, associ- ated with general stress response, contained multiple targets of the Msn2 and Msn4 stress TFs [37]. We validated that these motif enrichments are not a byproduct of the functional enrichment in the JACSs $\left(p<10^{-4}\right.$, by random sampling of gene groups with the same fraction of genes from the corresponding functional category as in the JACS). This analysis suggests that the JACS we obtained indeed correspond to gene modules with a common transcriptional regulation.

\section{Cell cycle in human}

We constructed a human protein-protein interaction network by combining information from the BIND and HPRD databases and from two recent large-scale yeast two-hybrid studies on human cells $[23,24]$. The resulting network contains 9,135 nodes and 25,086 interactions. Expression profiles of the synchronized HeLa cell lines from [38] were used. Only the 19 point time series obtained for synchronization by thymidine-nocodazole block was selected for the analysis, as it contains the fewest missing values. Genes for which the maximal fold change across the conditions was below 2 were filtered, leaving 1,536 genes (front nodes).
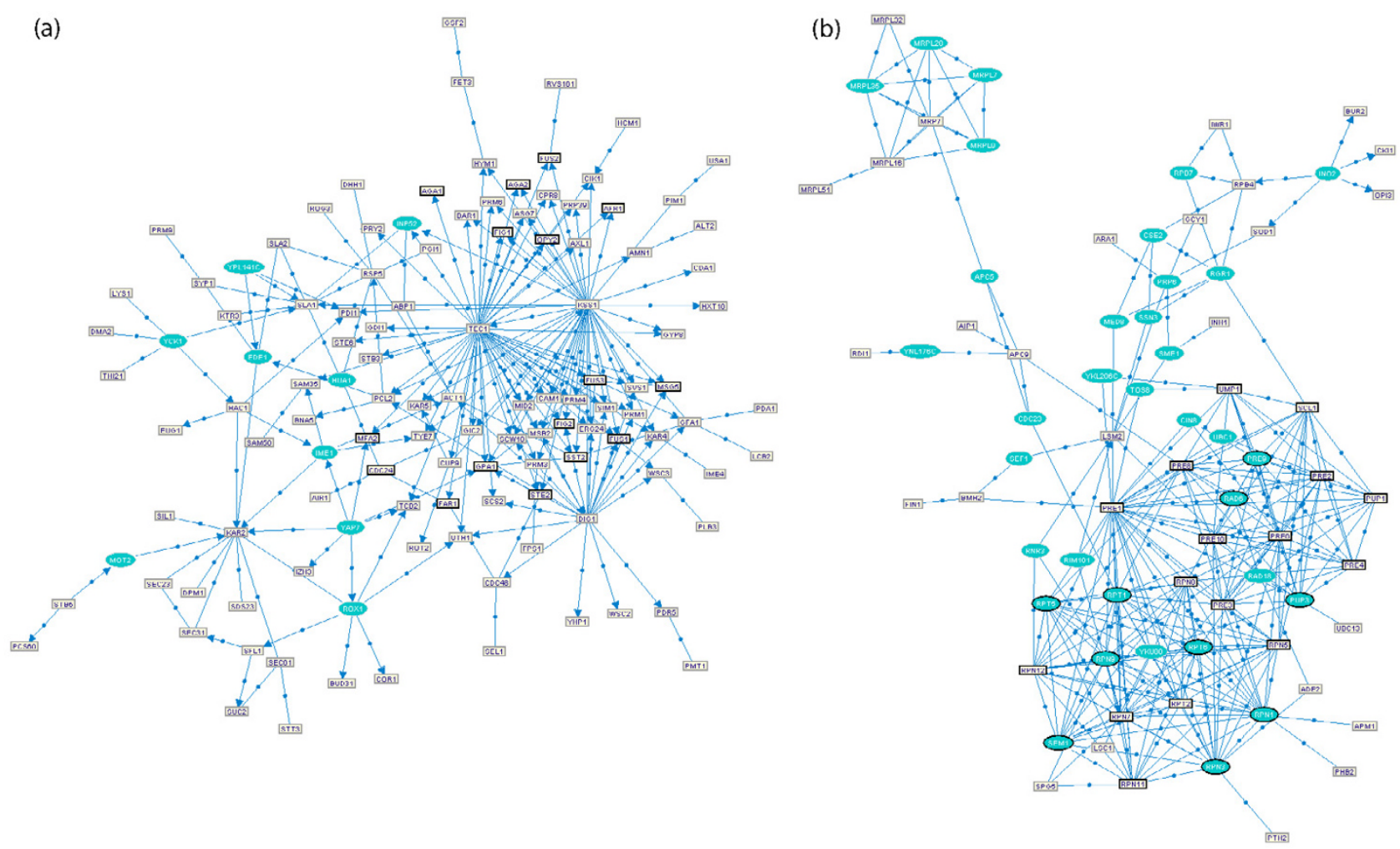

\section{Figure 4}

Two of the JACSs identified in the S. cerevisiae analysis. (a) The pheromone response subnetwork, (b) The proteolysis subnetwork. The front nodes are the yellow (light gray) rectangles and the back nodes and the blue (dark gray) ovals. The genes annotated with pheromone response (a) and proteolysis (b) are drawn with thicker border. Gene lists, expression matrices and interactive display of all the subnetworks are available at the supplementary website. 
We performed MATISSE analysis using the All-Neighbors heuristic, and the same parameters as in the previous section, and obtained 14 significant JACSs. Maps of these subnetworks are provided on our website and in the supplement [see Additional file 1]. To check the ability to discover subnetworks active at different cell cycle phases, we analyzed the overlap between the JACSs and annotations of specific cell-cycle phases as provided in [38]. Indeed, seven modules were enriched for specific phases of the cell cycle with $p<0.05$ after Bonferroni correction. The module with the highest cell cycle enrichment (JACS 5, $p=$ $2.85 \cdot 10^{-17}$ ) is shown in Figure 5a.

The advantage of MATISSE is evident when comparing the modules most enriched for the GO "cell cycle" category in the MATISSE and the Co-clustering solutions. While the MATISSE module is a single connected component of 120 genes, the corresponding co-cluster contains 110 connected components and 519 genes, and thus is much less amenable to interpretation in terms of the functional connections between its genes.

\section{Subnetwork hub analysis}

We hypothesized that the topology of the JACSs obtained by MATISSE can provide clues to the key players in the regulation of the cell cycle machinery. To test this, we looked for "subnetwork hubs" in the JACSs, i.e., genes whose degrees in a JACS were high both absolutely and relatively to their network degree (see Methods). This analysis on the 14 JACSs identified 52 hubs, 18 of them with "cell cycle" annotation $\left(p=5.13 \cdot 10^{-11}\right)$. This set contained many cell cycle master regulators such as p53, ATM, E2F1, $\mathrm{TGF} \beta \mathrm{R}, \mathrm{CDK} 4$ and CDC42. Remarkably, 36 out of 52 hubs form a single connected subnetwork, displayed in Figure 5b. This demonstrates that subnetwork hubs represent key regulators relevant to the experimental conditions tested. The interactions between the subnetwork hubs are putative regulatory interactions governing the progression of the cell cycle. As only 33 of the 52 hubs are

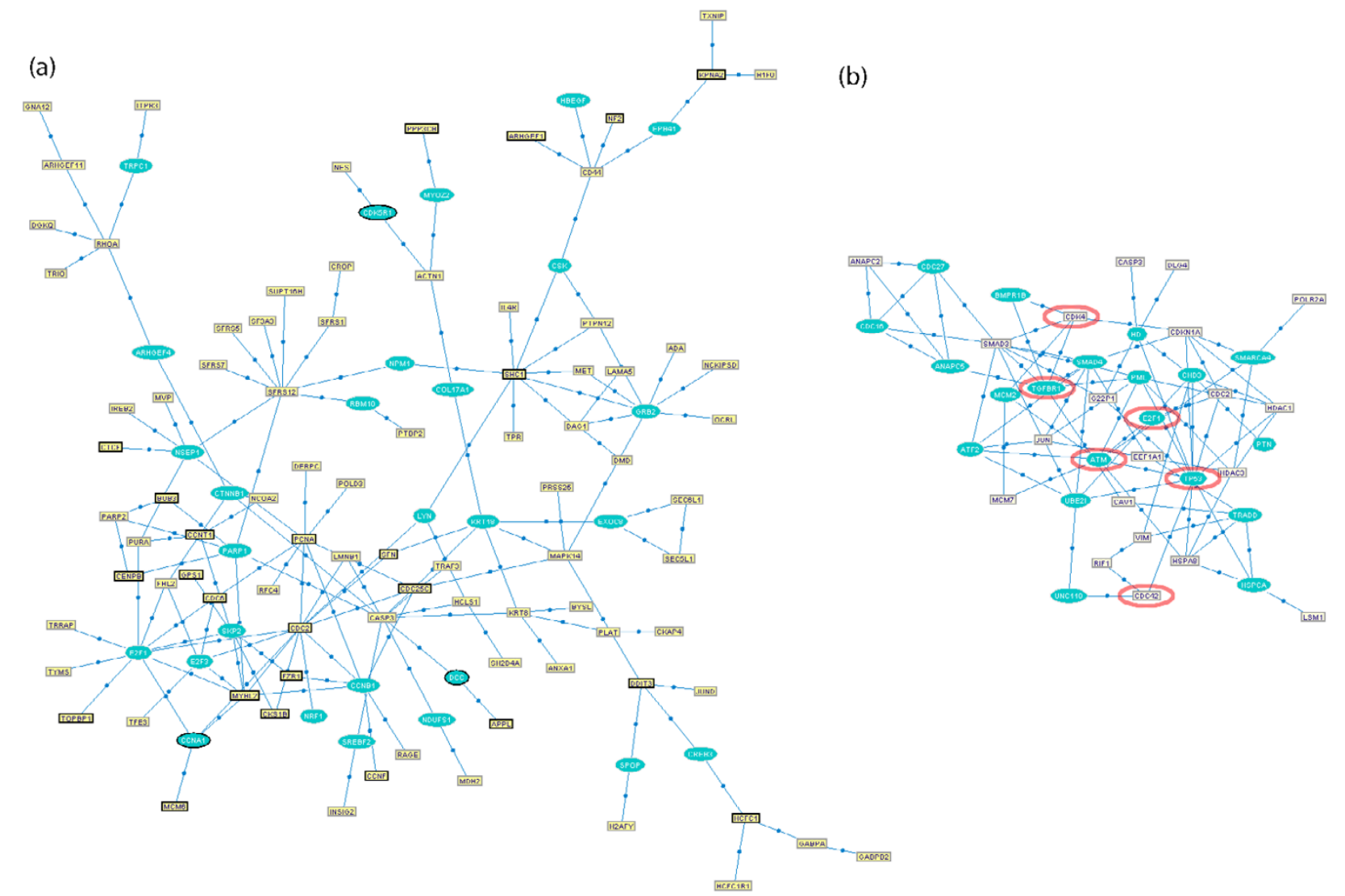

Figure 5

Examples of the MATISSE analysis in the cell cycle data of human HeLa cells. Front nodes and back nodes are as indicated in Figure 4. (a) The highest scoring cell-cycle related JACS identified. The genes annotated with "cell cycle" are drawn with thicker border. Gene lists, expression matrices and interactive display of all the subnetworks are available at the supplementary website, (b) Subnetwork hubs. The figure shows 36 nodes in the JACSs that were identified as subnetwork hubs and induced a connected component in the network. 16 additional hubs that had no interactions with other hubs are not shown. The known master regulators p53, ATM, E2FI, TGF $\beta$ R, CDK4 and CDC42 are circled. 
front nodes in their respective JACS, this set could not be identified using expression data alone.

\section{Conclusion}

We have developed a novel computational technique for the integrated analysis of network and similarity data. The method is aimed to dissect together topological properties of gene or protein networks and other high-throughput data. We used the method to analyze large-scale protein interaction networks and genome-wide transcription profiles in yeast and human. The method was shown to identify functionally sound modules, i.e., connected subnetworks with highly coherent expression showing significant functional enrichment. In comparison to the extant Co-clustering method, which aims to integrate similar data, our method demonstrated substantial improvement in solution quality. Comparison to solutions produced by clustering highlights the advantage of utilizing topological connectivity in the hunt for functionally sound modules. By construction, our method is specifically powerful in detection of regulatory modules, and less fit for detection of metabolic modules. Our technique, implemented in the program MATISSE, is efficient and can analyze genome-scale interaction and expression data within minutes.

The proposed algorithm is very flexible and - unlike Coclustering - can handle situations where not all genes in the network have similarity information or expression patterns. In particular, MATISSE can determine the subset on which similarity is computed using various criteria, e.g., initial probe filtering, differential expression confidence values, etc. As we demonstrate, even when only a modest fraction of the overall network genes have expression/similarity information, the method finds meaningful modules successfully.

The requirement for network connectivity as proposed in our method can be viewed as problematic due to high rate of false negative interactions. A natural extension of MATISSE which we intend to pursue is to take into account the interaction confidence. As a first step towards this goal, we assessed the composition of the interactions in the reported subnetworks as follows: we compared the observed and expected number of interactions within the subnetworks, from each of the publications used as interaction sources in the S. cerevisiae interactions network. We found a clear enrichment for interactions from recent experiments, such as [39] and [40], opposed to an underrepresentation of interactions from older works, such as $[41,42]$ and [43] (see supplementary table). As currently the coverage of the protein interaction network is limited, we suggest performing MATISSE analysis in addition to standard clustering analysis.
The framework described in this work is directly applicable to any kind of pairwise similarity data where the probabilistic assumptions hold. While this study focused on protein interaction networks and gene expression, the approach is general enough to treat many other data types. These include other types of interactions, such as genetic interactions, regulation and protein-DNA binding patterns, and other similarity measures, such as functional similarity or similarity in protein-DNA binding profiles [2]. We intend to extend MATISSE to these types of data as well.

While the rapidly expanding resource of microarray data is currently analyzed primarily using diverse clustering techniques, methods for the analysis of network-type data describing interrelations of genes and proteins are less mature, and methods for joint analysis of the two data types are in nascent stage. We expect the proposed method to become widely used for dissecting expression data in light of the interaction knowledge. Our initial results show that despite the high complexity and the relatively low coverage of the human interactome, biologically relevant modules can be found in the human protein interaction network through integrative analysis.

\section{Methods \\ The probabilistic model}

Recall that we formalize the problem as finding disjoint node sets that induce connected subgraphs in the constraint graph and manifest high internal similarity. We formulate this problem as a hypothesis testing question. For this, we define a probabilistic model for the similarity data, using ideas from [27] and [44]. Given a set $U$ of $k$ genes, we compare two hypotheses: the null hypothesis $H_{0}$ : $U$ is a set of unrelated genes; and the JACS hypothesis $H_{1}: U$ is a JACS. We assume that the observed pairwise similarity values are a mixture of two Gaussian distributions: one for pairs of genes that are highly co-expressed (such pairs are called mates) and another for the rest. Let $M_{i j}$ denote the event that $i$ and $j$ are mates. The similarity values between mates $\left(P\left(S_{i j} \mid M_{i j}\right)\right)$ are normally distributed with mean $\mu_{m}$ and variance $\sigma_{m}^{2}$. The similarity levels of all non-mates are distributed normally with the parameters $\mu_{n}$ and $\sigma_{n}^{2}$. These assumptions are theoretically justified in certain situations [27]. Empirically, analysis using normal quantile plots [45] indicates that they are valid for the biological data analyzed in this paper (results not shown). We also assume that the probability that a pair of genes are mates is high if they belong to the same JACS and low otherwise. 


\section{Differential regulation}

Not all genes within the interaction network are regulated on the expression level. Thus, when working with expression profiles, we would like the model to allow lower similarity levels between genes that are not necessarily regulated on the expression level, while penalizing heavily for low similarity between transcriptionally regulated genes. This allows flexibility on two levels in our setting. First, the genes can be filtered prior to computing similarities (e.g., only genes passing a threshold of observed fold change or variation level are included in $V_{\text {sim }}$ ). Note that genes that fail to pass the filter remain in the interaction network and can be incorporated into a JACS, while not used for its scoring. Second, a prior can be assigned to the likelihood that a gene is regulated: we define $R_{i}$ as the event that gene $i$ is regulated on the expression level under the conditions studied and let $P\left(R_{i}\right)$ designate the probability of that event.

\section{The likelihood score}

We assume that JACSs contain a much higher proportion of mates than gene pairs that do not belong to the same JACS. Specifically, we assume that a large fraction $\beta_{m}$ (e.g. $0.9)$ of the pairs of transcriptionally regulated genes within the JACS are mates and thus their similarity levels are distributed $N\left(\mu_{m}, \sigma_{m}\right)$. Then $P\left(M_{i j} \mid R_{i} \wedge R_{j}, H_{1}\right)=\beta_{m}$. We make the simplifying approximation that the scores of different gene pairs are independent. Consequently, the likelihood of a JACS $U$ is decomposable on every pair of genes in it:

$$
P\left(S_{U \times U} \mid H_{1}\right)=\prod_{(i, j) \in U \times U} P\left(S_{i j} \mid H_{1}\right)
$$

Let $\gamma_{i j}^{m}=\beta_{m} P\left(R_{i}\right) P\left(R_{j}\right)$. Then:

$$
P\left(S_{i j} \mid H_{1}\right)=\gamma_{i j}^{m} P\left(S_{i j} \mid M_{i j}\right)+\left(1-\gamma_{i j}^{m}\right) P\left(S_{i j} \mid \overline{M_{i j}}\right)
$$

The null hypothesis $\left(H_{0}\right)$ is that the fraction of mates in $U$ is not surprising: every two transcriptionally regulated genes are mates with the probability expected from the relative portion of mates among all the regulated genes, denoted $p_{m}$. Let $\gamma_{i j}^{n}=p_{m} P\left(R_{i}\right) P\left(R_{j}\right)$. The likelihood ratio between the two hypotheses $\left(\frac{P\left(\text { Data } \mid H_{1}\right)}{P\left(\text { Data } \mid H_{0}\right)}\right)$ is:

$$
\frac{\prod_{(i, j) \in U \times U} \gamma_{i j}^{m} P\left(S_{i j} \mid M_{i j}\right)+\left(1-\gamma_{i j}^{m}\right) P\left(S_{i j} \mid \overline{M_{i j}}\right)}{\prod_{(i, j) \in U \times U} \gamma_{i j}^{n} P\left(S_{i j} \mid M_{i j}\right)+\left(1-\gamma_{i j}^{n}\right) P\left(S_{i j} \mid \overline{M_{i j}}\right)}=\prod_{(i, j) \in U \times U} \frac{\gamma_{i j}^{m} P\left(S_{i j} \mid M_{i j}\right)+\left(1-\gamma_{i j}^{m}\right) P\left(S_{i j} \mid \overline{M_{i j}}\right)}{\gamma_{i j}^{n} P\left(S_{i j} \mid M_{i j}\right)+\left(1-\gamma_{i j}^{n}\right) P\left(S_{i j} \mid \overline{M_{i j}}\right)}
$$

Define the similarity graph, $G^{S}=\left(V_{\text {sim }} E^{S}\right)$, where $E^{S}=\left(V_{\text {sim }}\right.$ $\left.\times \quad V_{\text {sim }}\right)$ and set

$$
\left.V_{\text {sim }}\right)
$$$$
\text { and }
$$

$w_{i j}=\log \frac{\gamma_{i j}^{m} P\left(S_{i j} \mid M_{i j}\right)+\left(1-\gamma_{i j}^{m}\right) P\left(S_{i j} \mid \overline{M_{i j}}\right)}{\gamma_{i j}^{n} P\left(S_{i j} \mid M_{i j}\right)+\left(1-\gamma_{i j}^{n}\right) P\left(S_{i j} \mid \overline{M_{i j}}\right)}$ as the weight of the edge $\left(v_{i}, v_{j}\right)$. The log-likelihood score for a given $U$ translates to the total edge weight of the subgraph induced by $U$ in $G^{S}$.

\section{JACS finding algorithm}

Our goal is to find disjoint sets $U_{1}, U_{2}, \ldots, U_{m}$ that induce connected subgraphs in $G^{C}$ and heavy subgraphs in $G^{S}$. When weights can be both positive and negative (as is the case in our formulation), even the problem of finding a single heavy subgraph is NP-Hard (by a simple reduction from Max-Clique using a complete constraint graph). Hence, exact optimization is intractable, and we experimented with several heuristic algorithms for solving the problem. All the schemes share the following three phases: (1) detection of relatively small, high-scoring gene sets, or seeds, (2) seed improvement, and (3) significancebased filtering.

\section{Identifying seeds}

We tested three different methods for generating high scoring seeds. In all the methods a large set of non-overlapping potential seeds is first generated, and only seeds passing a certain score threshold are passed to the next phase.

\section{Best-neighbors}

In this method, high scoring seeds of a predefined size $k$ are constructed. The nodes of the graph are ranked based on their total incident edge weights in $G^{S}$ (their weighted degree). The algorithm repeatedly creates a seed and removes its nodes from the graph. The seed generating step picks the highest ranking node $v$, and selects a set of $k$ - 1 neighbors of $v$ in $G^{S}$ that maximize the seed score. The optimal neighbor set can be found through exhaustive enumeration (enumeration is needed since the score for different neighbor sets depends also on the weights of the edges between them). When enumeration is computationally prohibitive, a heuristic that picks nodes with the highest weighted degree within the immediate neighborhood of $v$ is utilized. Specifically, let $N_{v}$ be the set of all the immediate neighbors of $v$. For $i \in N_{v}$ define $w_{i}^{v}=\sum_{v_{j} \in N_{v}} w_{i j}$. The heuristic selects $k-1$ nodes with the highest $w^{v}$ values.

\section{All-neighbors}

This method is similar to Best-Neighbors, but instead of selecting $k$ - 1 neighbors for a potential seed, in this version, all the neighbors of $v$ with a non-negative edge score (including neighboring back nodes with zero score) enter the seed. 


\section{Heaviest-subnet}

This method is inspired by Charikar's 2-approximation algorithm for the densest subgraph problem [46]. An articulation node in a connected graph is one whose removal disconnects the graph. The following algorithm is executed independently on each connected component in the constraint graph. The algorithm works in a "destructive" fashion: starting from the original constraint graph, nodes are removed from the graph one at a time until none remain. The next node to be removed is one with the smallest weighted degree in the current similarity graph that is not an articulation node in the current constraint graph. It is easy to see that such a node always exists. After each node removal, the overall score of the remaining graph is recorded. After all nodes are removed, the highest-scoring (possibly size-constrained) subgraph that was encountered is selected as the seed. That subgraph is then removed from the graph and the next seed is sought.

\section{Seed optimization}

Once a set of high-scoring seeds is established, a greedy algorithm aims to optimize all the seeds simultaneously. In our tests, this strategy worked better than optimizing each seed separately, as it produced more diverse JACSs. The algorithm keeps a set of disjoint subnetworks at every iteration and considers the following moves (Figure 6):

\section{Node addition}

Addition of an unassigned node to an existing JACS.

Node removal

Removal of a node from a JACS.

\section{Assignment change}

Exchange of a node between JACSs.

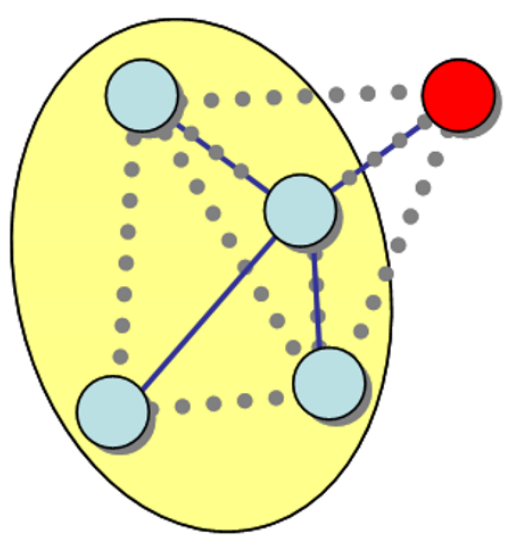

(a)

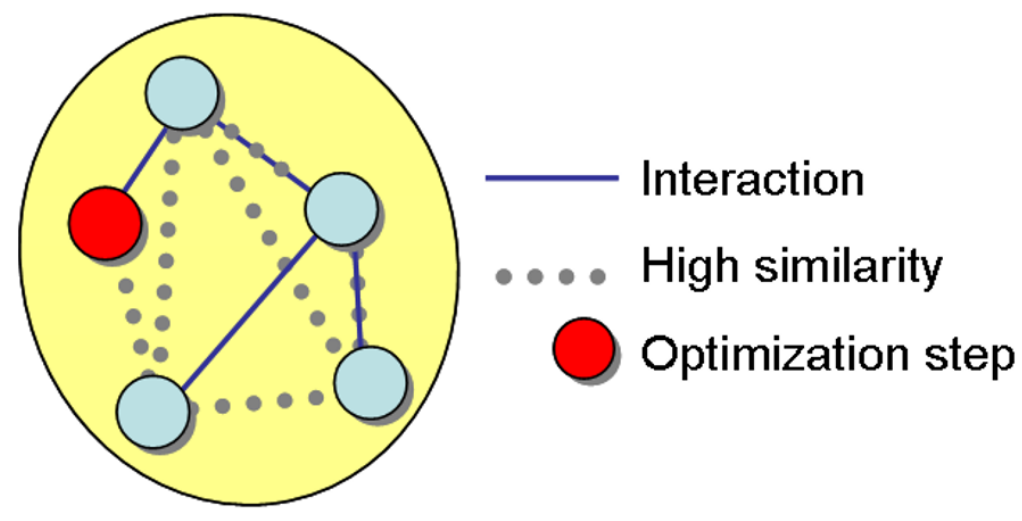

(b)

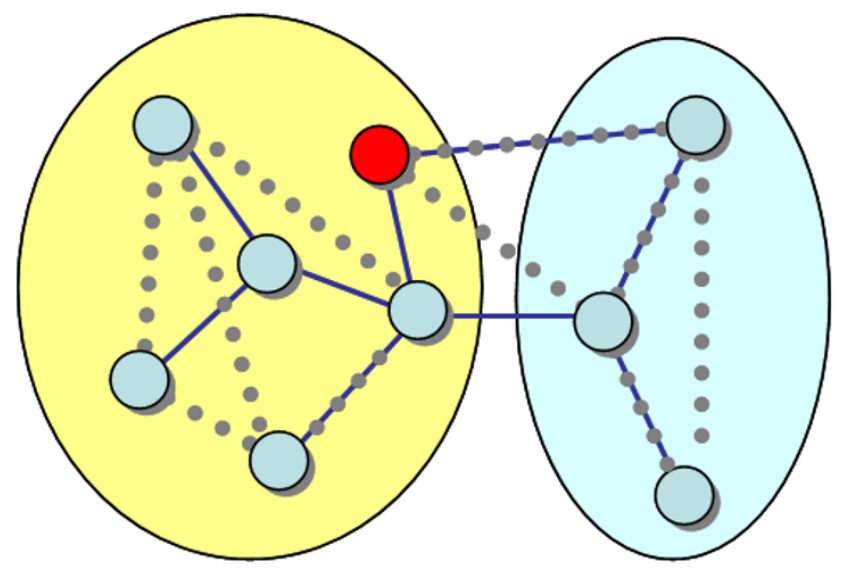

(c)

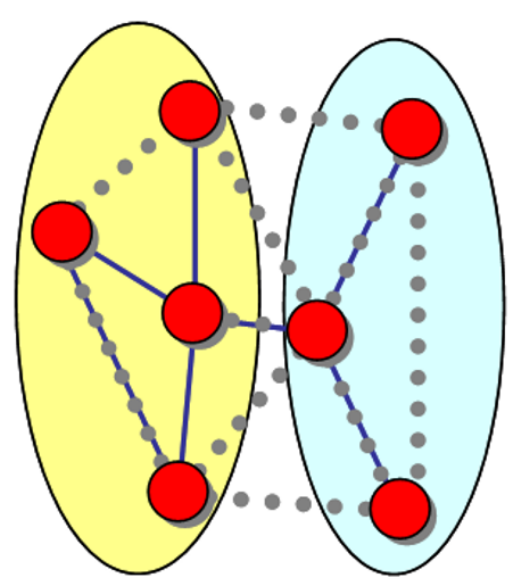

(d)

\section{Figure 6}

Toy examples of the moves performed by the optimization algorithm. (a) Node addition; (b) Node removal; (c) Assignment change; (d) JACS merge. In each case the affected nodes are in red (black). 


\section{JACS merge}

A new JACS is formed by taking the union of the nodes in two existing JACSs. This step is particularly beneficial when the original seeds are relatively small.

At every step a move is selected only if (1) it improves the overall score of the solution, i.e., the sum of the weights of all the JACSs and (2) the move maintains the connectivity of the JACSs. If no such step exists, a "cleanup" procedure iteratively removes from every JACS non-articulation back nodes that are not found on any simple path between front nodes. If the clean-up step does not remove any nodes, the optimization halts. Note that the algorithm is guaranteed to converge, as the global score is monotonically increasing. In addition, in order to obtain biologically meaningful JACSs, an upper bound on the size of a JACS can be employed throughout the optimization. If a JACS reaches this upper bound in the course of the optimization, any node added to it causes a removal of a lowscoring node, maintaining the JACS size. Note that this procedure can add only front nodes.

\section{Filtering}

After a collection of putative JACSs is obtained, it is filtered based on the significance of the JACS score. For that purpose, for every candidate JACS, an empirical p-value of its score is calculated using sampling randomly gene groups of the same size. Only candidate JACSs with pvalue below a threshold $p$ pass the filtering stage $(p=0.05$ after Bonferroni correction was used). In a second step, to avoid possible bias in the score, we empirically test the JACS significance using only expression similarity scores. The same sampling procedure is performed using the average raw expression pairwise similarity values, and JACSs whose average similarity is not sufficiently high compared to the sampled sets of the same size are removed. An efficient computation of this step is done as suggested in [15].

\section{Implementation issues}

For efficient implementation, several slight modifications were made to the algorithm described above:

\section{Removal of non-contributing nodes}

As in our framework only front nodes are used for JACS scoring, back nodes will be incorporated into the subnetwork only if they appear on some path between two front nodes. Thus, prior to algorithm execution we remove from $G^{C}$ all back nodes that are leaves (nodes with degree smaller than 2). The procedure is iterated until no such leaves remain in the graph. In practice, due to the nature of the protein interaction network used, this step significantly reduces the size of the network, without influencing the quality of the solution.

\section{Similarity graph adjustment}

When finding Heaviest-Subnet seeds, low edge density in the graph is crucial for efficiency. We therefore remove edges with low absolute weight from the graph, as their contribution to the overall JACS score is small. All the edges are used in the subsequent phases.

\section{Finding heaviest-subnet seeds}

Efficient implementation of this algorithm can be done using a data structure similar to the one developed for the dynamic connectivity problem [47]. This would take $O\left(|V| \log ^{4}|V|\right)$ time per seed. Instead, we used a simple algorithm for detection of articulation nodes in each iteration. Articulation nodes can be detected during a depthfirst traversal of the graph, by calculating the "lowpoint" values of every node (cf. [48]).

This implementation required complexity of $O\left(|V|\left|E^{S}\right|\right)$ time per seed. Since this time can be too long for very large graphs, we use a sampling approach when the component contains more than 1,500 nodes: a connected subgraph of a more modest size is randomly sampled (as described in [49]) and then used for seed finding. This sampling is repeated several times, with the highest scoring seed used for further optimization.

\section{Implementation}

MATISSE was implemented as a Java stand-alone application. In addition to the algorithmic engine, it contains a visualization tool allowing flexible inspection of the obtained subnetworks and diverse post-process analyses. Running times are efficient enough to accommodate large interaction networks and gene expression datasets. For example, on a constraint graph of 4, 543 nodes and 1,996 expression profiles, the processing took less than $15 \mathrm{~min}$ utes for All-Neighbors and Best-Neighbors methods and 78 minutes for Heaviest-Subnet, on a Pentium $43 \mathrm{GHz}$ machine with 2 GB memory. About $10-20 \%$ of the time is needed to learn the parameters using EM, and this time is saved in all subsequent runs on the same data. The running time depends sublinearly on the bound on the maximum size of the JACS (Figure 7). The application will soon be available at [50].

\section{Simulation setup}

Our simulations used the real connected network of 2,000 yeast proteins described in Results, and synthetic similarity values, generated as follows. First, a set of $m$ disjoint connected subnetworks $P_{1}, \ldots, P_{m}$ of equal size $k$ was randomly selected as in [49]. Then, from each subnetwork a subset of size $k \cdot p_{f}$ was randomly selected to be included in $V_{\text {sim }}$ (front nodes). The resulting $V_{\text {sim }}$ was expanded by additional randomly selected nodes, to contain $n_{\text {sim }}$ nodes in total. Similarity values were generated as in [27] using two Gaussian distributions - $N_{m}$ with parameters $\mu_{m^{\prime}} \sigma_{m}$ for similarity between mates and $N_{n}$ with parameters $\mu_{n}$ $\sigma_{n}$ for all other pairs.

Similarity values were determined independently for each node pair, as follows: If the two nodes reside in the same JACS, the value was drawn from $N_{m}$ with probability $\beta_{m}$ 


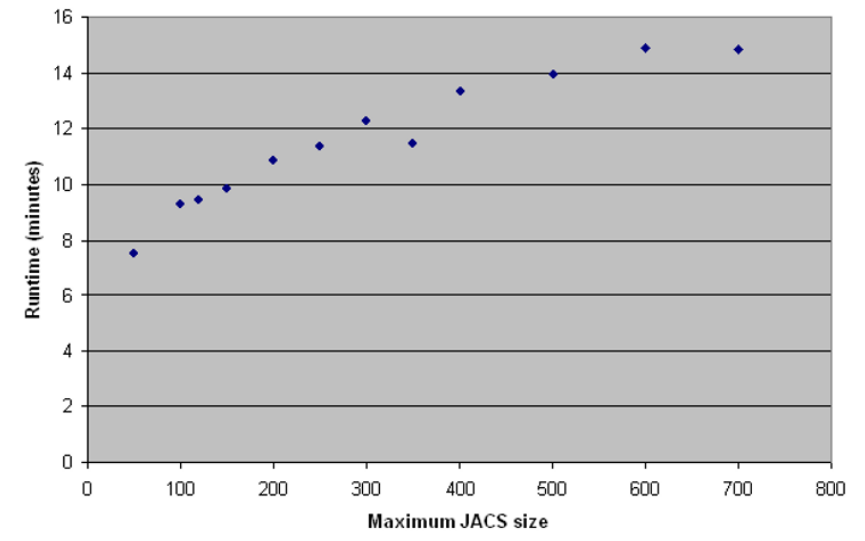

Figure 7

Dependence of the running time on the size of the JACS. The running time of MATISSE with different maximum JACS size parameters. The execution did not include the weight calculation step, as it is not dependent on the JACS size.

and from $N_{n}$ with probability $1-\beta_{m}$. Otherwise, the value was drawn from $N_{m}$ with probability $p_{m}$.

The default values for the simulations were set to $n_{\text {sim }}=1$, 000 (out of $|V|=2$, 000);

$m=6 ; k=100 ; p_{f}=0.7 ; \mu_{m}=0.5 ; \mu_{n}=0 ; \sigma_{m}=\sigma_{n}=0.3 ; \beta_{m}=$ $0.95 ; p_{m}=0.01$.

\section{Evaluating performance}

The success of an algorithm in recovering the planted components was measured using the Jaccard coefficient [51]. It is defined as $\frac{n_{11}}{n_{11}+n_{10}+n_{01}}$, where $n_{11}$ is the number of node pairs included both in the same planted component and in the same JACS, $n_{10}$ is the number of pairs included in the same planted component but not in the same JACS, and $n_{01}$ is the number of pairs in the same JACS but not in the same planted component. Hence, a perfect fit of the two solutions would get a score of 1 , and lower scores indicate reduced fit.

\section{Parameter estimation}

To obtain meaningful results, a good assessment of the parameters of the probabilistic model is prerequisite. We tested different schemes for assessing $P\left(R_{i}\right)$, and selected the following scheme. We ranked the genes based on the variation observed across their expression patterns and then applied a logistic function to the normalized ranks to obtain: $P\left(R_{i}\right)=\alpha+(1-\alpha) \frac{1}{1+e^{-\beta\left(x_{i}-\gamma\right)}}$, where $x_{i}$ is the normalized rank of gene $i$. The logistic parameters were empirically set to $\alpha=0.6, \beta=24$ and $\gamma=0.25$. To evaluate the effect of the specific form of the prior on the results, we reran the JACS finding algorithms with different logistic parameter settings ( $\alpha=0.4 . .0 .8, \beta=1 . .24, \gamma=0.2 . .0 .7$ ). The average expression homogeneity and the average functional homogeneity of the produced JACSs (computed as described in [1]) of the JACSs did not change by more than $6 \%$.

We adjusted the standard EM algorithm used for learning a mixture of Gaussians (cf. [52]) in order to estimate $\mu_{m^{\prime}}$ $\sigma_{n^{\prime}} \mu_{n^{\prime}} \sigma_{n}$ and $p_{m}$. A detailed description of the EM algorithm can be found at our website ([50]). The produced JACSs were constrained to the size range of 5-120 and $\beta_{m}$ was set to 0.9 . We verified that the reported results are robust to changes in the value of $\beta_{m}$ by varying it between 0.75 and 0.99 and analyzing the obtained solutions. We found that both the average expression homogeneity and the average functional homogeneity did not change by more than 3\% across this parameter range.

\section{Comparison of the heuristics}

We evaluated the three proposed heuristics both in our simulation setting and on the osmotic shock response in $S$. cerevisiae. The results of the comparison on simulation data are presented in Figure 8 . Overall, as can be seen in Figure 8, all three MATISSE variants show similar performance. All the methods exhibit poor performance in detection of small planted components $(k<50)$. BestNeighbors seems to be the preferred method on the simulated data. Best-Neighbors and All-Neighbors is that Best-Neighbors does not incorporate back nodes at all, while All-Neighbors may include some. As we shall show below, using back nodes is in fact advantageous in real biological data. The performance of the Heaviest-Subnet seeding is highly variable, probably due to its relatively significant dependency on the structure of the similarity graph.

The results of the comparison on simulation data are presented in Figure 9. The Best-Neighbors variant performs slightly better than All-Neighbors in terms of the fraction of enriched modules, but All-Neighbors performs significantly better in terms of category coverage, due to its inclusion of back nodes. We therefore carried out all subsequent analysis using the modules produced with the All-Neighbors variant.

\section{Functional enrichment analysis}

We used the TANGO algorithm [31] for finding GO terms enriched in the JACSs. The algorithm considers all levels of GO and corrects p-values for multiple testing and for category dependency using resampling. Briefly, TANGO repeatedly selects random sets of genes to compute an 
(a)

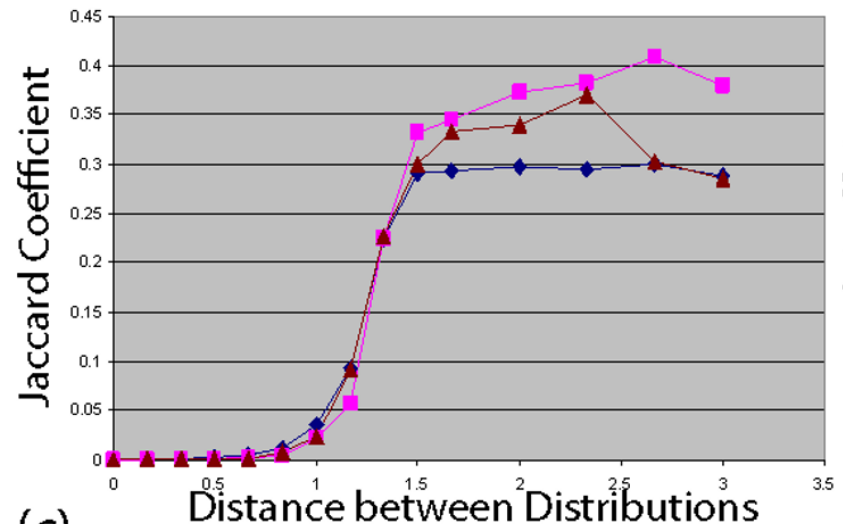

(c)

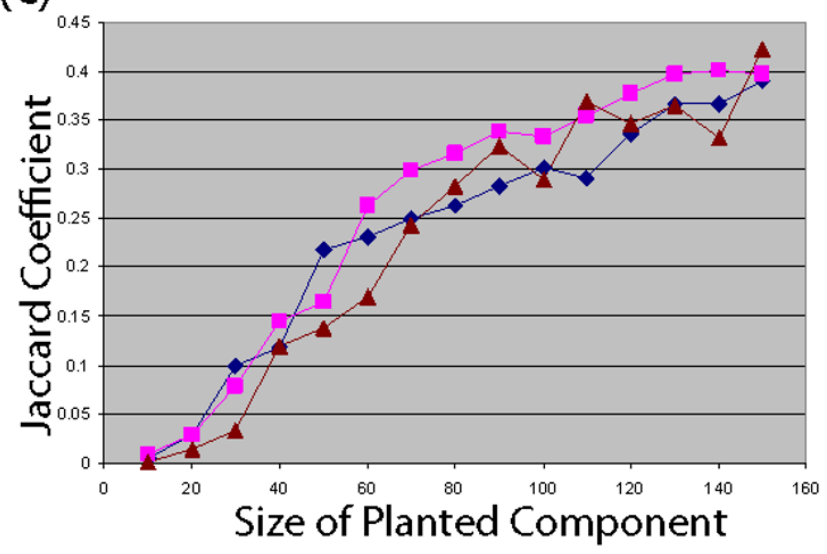

(b)

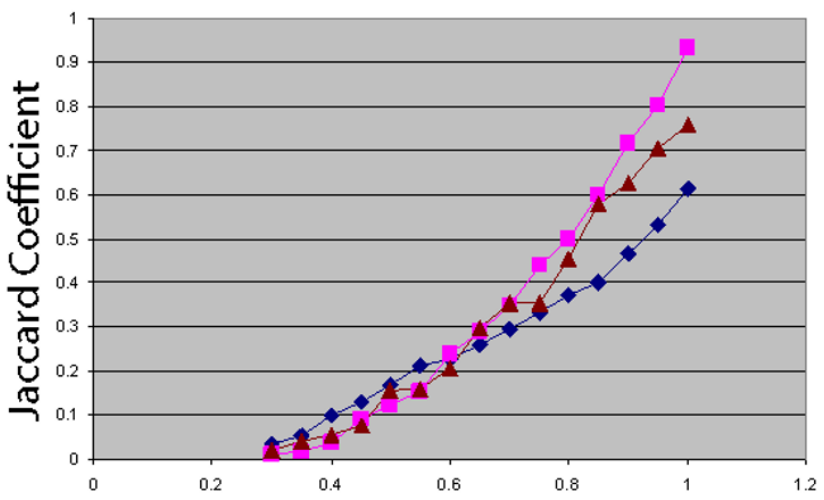

Fraction of Front Nodes

Figure 8

Performance of the three proposed heuristics on simulated data. See Figure 2 for further details.

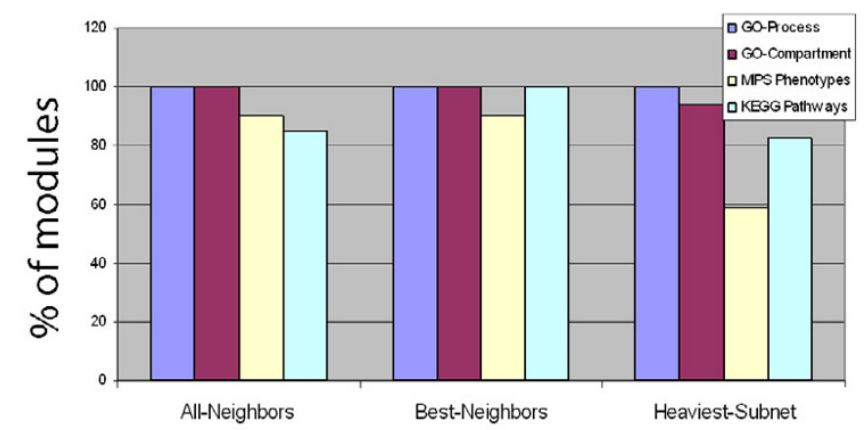

(a)

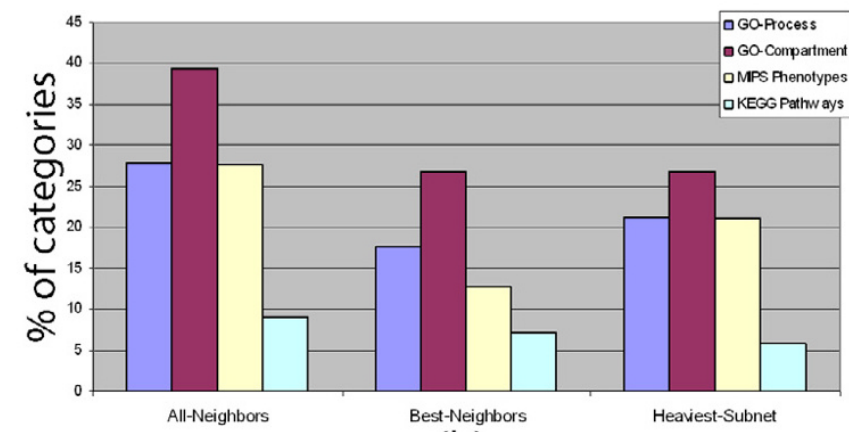

(b)

Figure 9

Performance of the three proposed heuristic in terms of annotation enrichment. See Figure 3 for further details. 
empirical distribution of maximum p-values for functional enrichment obtained across a random sample of sets that maintain the same size characteristics of the ones analyzed. TANGO uses this empirical distribution to determine thresholds for significant enrichment on the true clusters. The algorithm filters out redundant categories by performing conditional enrichment tests that ensure that all the reported enriched categories are statistically significant even after taking into account the enrichment of their ancestor and children nodes in the tree.

\section{Extraction of subnetwork hubs}

Given a JACS $J, v \in J$ was called a hub if it satisfied three requirements: (a) the degree of $v$ within the subnetwork $J$ exceeds 7 ; (b) the degree of $v$ in $J$ is among the five highest in $J$; (c) the degree of $v$ in $J$ is significantly high given its degree in the whole network $(p<0.05$ using hypergeometric distribution). Note that back nodes can also be hubs.

\section{Authors' contributions}

IU and RS designed the study. IU developed MATISSE and performed the statistical analysis. IU and RS wrote the manuscript. Both authors read and approved the final manuscript.

\section{Additional material}

\section{Additional File 1}

JACSs identified by MATISSE. Images of the subnetworks identified by MATISSE in the osmotic shock response and the cell cycle datasets. Click here for file

[http://www.biomedcentral.com/content/supplementary/17520509-1-8-S1.pdf]

\section{Acknowledgements}

We thank Irit Gat-Viks, Chaim Linhart, Daniela Raijman, Israel Steinfeld and Amos Tanay for helpful discussions. IU is supported in part by a fellowship from the Safra Foundation. RS was supported in part by the Wolfson foundation, and by the EMI-CD project that is funded by the European Commission within its FP6 Programme, under the thematic area "Life Sciences, genomics and biotechnology for health", contract number LSHG-CT-2003503269.

\section{References}

I. Lord P, Stevens R, Brass A, Goble C: Investigating semantic similarity measures across the Gene Ontology: the relationship between sequence and annotation. Bioinformatics 2003, 19(10): 1275-83

2. Kim R, Ji J, Wong W: An improved distance measure between the expression profiles linking co-expression and co-regulation in mouse. BMC Bioinformatics 2006, 7:44.

3. Ge H, Liu Z, Church G, Vidal M: Correlation between transcriptome and interactome mapping data from Saccharomyces cerevisiae. Nat Genet 200I, 29(4):482-486.

4. Hahn A, Rahnenführer J, Talwar P, Lengauer T: Confirmation of human protein interaction data by human expression data. BMC Bioinformatics 2005, 6: I I 2 .

5. Jansen R, Yu H, Greenbaum D, Kluger Y, Krogan NJ, Chung S, Emili A, Snyder M, Greenblatt JF, Gerstein M: A Bayesian networks approach for predicting protein-protein interactions from genomic data. Science 2003, 302(5644):

6. de Lichtenberg U, Jensen L, Brunak S, Bork P: Dynamic complex formation during the yeast cell cycle. Science 2005, 307(57 I0):

7. Luscombe N, Babu M, Yu H, Snyder M, Teichmann S, Gerstein M Genomic analysis of regulatory network dynamics reveals large topological changes. Nature 2004, 43 I(7006):

8. Wachi S, Yoneda K, Wu R: Interactome-transcriptome analysis reveals the high centrality of genes differentially expressed in lung cancer tissues. Bioinformatics 2005, 21 (23):4205-4208.

9. Balazsi G, Barabasi A, Olvai Z: Topological units of environmental signal processing in the transcriptional regulatory network of Escherichia coli. PNAS 2005, 102(22):784I-7846.

I0. van Helden J, Gilbert D, Wernisch L, Schroeder M, Wodak S: Application of Regulatory Sequence Analysis and Metabolic Network Analysis to the Interpretation of Gene Expression Data. In Proc JOBIM '00 London, UK: Springer-Verlag; 2000:147-I64.

II. Hartwell LH, Hopfield JJ, Leibler S, Murray AW: From molecular to modular cell biology. Nature 1999, 402(676I Suppl):.

12. Zien A, Kuffner R, Zimmer R, Lengauer T: Analysis of Gene Expression Data with Pathway Scores. Proc ISMB '00 2000:407-4I7.

13. Kurhekar M, Adak S, Jhunjhunwala S, Raghupathy K: Genome-wide pathway analysis and visualization using gene expression data. In Proc PSB '02 Springer-Verlag; 2002:462-73

14. Kanehisa M, Goto S: KEGG: Kyoto encyclopedia of genes and genomes. Nucleic Acids Res 2000, 28:27-30.

15. Jansen R, Greenbaum D, Gerstein M: Relating whole-genome expression data with protein-protein interactions. Genome Research 2002, I 2:37-46.

16. Vert J, Kanehisa M: Extracting active pathways from gene expression data. Bioinformatics 2003, 19:||238-I|244.

17. Hanisch D, Zien A, Zimmer R, Lengauer T: Co-clustering of biological networks and gene expression data. Bioinformatics 2002 , I8:SI 45-54.

18. Eisen MB, Spellman PT, Brown PO, Botstein D: Cluster analysis and display of genome-wide expression patterns. PNAS 1998 , 95:| $4863-14868$

19. Ideker T, Ozier O, Schwikowski B, Siegel AF: Discovering regulatory and signalling circuits in molecular interaction networks. Bioinformatics 2002, I 8:S233-S240.

20. Cabusora L, Sutton E, Fulmer A, Forst C: Differential network expression during drug and stress response. Bioinformatics 2005, 2 I ( I 2):2898-2905

21. Segal E, Wang H, Koller D: Discovering molecular pathways from protein interaction and gene expression data. Bioinformatics 2003, I9 Suppl I:i264-7I.

22. Ihmels J, Levy R, Barkai N: Principles of transcriptional control in the metabolic network of Saccharomyces cerevisiae. Nat Biotechnol 2003, 22:86-92.

23. Stelzl U, Worm U, Lalowski M, Haenig C, Brembeck FH, Goehler H, Stroedicke M, Zenkner M, Schoenherr A, Koeppen S, Timm J, Mintzlaff S, Abraham C, Bock N, Kietzmann S, Goedde A, Toksoz E, Droege A, Krobitsch S, Korn B, Birchmeier W, Lehrach H, Wanker $\mathrm{EE}$ : A human protein-protein interaction network: a resource for annotating the proteome. Cell 2005, I 22(6):957-968.

24. Rual JF, Venkatesan K, Hao T, Hirozane-Kishikawa T, Dricot A, Li N, Berriz GF, Gibbons FD, Dreze M, Ayivi-Guedehoussou N, Klitgord N, Simon C, Boxem M, Milstein S, Rosenberg J, Goldberg DS, Zhang LV Wong SL, Franklin G, Li S, Albala JS, Lim J, Fraughton C, Llamosas E, Cevik S, Bex C, Lamesch P, Sikorski RS, Vandenhaute J, Zoghbi HY, Smolyar A, Bosak S, Sequerra R, Doucette-Stamm L, Cusick ME, Hill $D E$, Roth FP, Vidal M: Towards a proteome-scale map of the human protein-protein interaction network. Nature 2005, 437(7062): $1173-1178$.

25. Ptacek J, Devgan G, Michaud G, Zhu H, Zhu X, Fasolo J, Guo H, Jona G, Breitkreutz A, Sopko R, McCartney RE, Schmidt MC, Rachidi N, Lee SJ, Mah AS, Meng L, Stark MJ, Stern DF, De Virgilio C, Tyers M, Andrews B, Gerstein M, Schweitzer B, Predki PF, Snyder M: Global analysis of protein phosphorylation in yeast. Nature 2005, 438(7068):679-84.

26. O'Rourke S, Herskowitz I: Unique and redundant roles for Hog MAPK pathway components as revealed by whole-genome expression analysis. Mol Biol Cell 2004, I5:532-42.

27. Sharan R, Shamir R: CLICK: A clustering algorithm with applications to gene expression analysis. In Proc Int Conf Intell Syst Mol Biol Volume 8. AAAI Press; 2000:307-316. 
28. Watts DJ, Strogatz SH: Collective dynamics of 'small-world' networks. Nature 1998, 393(6684):440-442.

29. Ashburner M, Ball C, Blake J, Botstein D, Butler H, Cherry J, Davis A, Dolinski K, Dwight S, Jea Eppig: Gene ontology: Tool for the unification of biology. Nature Genetics 2000, 25:25-29.

30. Mewes HW, Frishman D, Guldener U, Mannhaupt G, Mayer K, Mokrejs M, Morgenstern B, Munsterkotter M, Rudd S, Weil B: MIPS: a database for genomes and protein sequences. Nucleic Acids Res 2002, 30(I):3।-4.

31. Shamir R, Maron-Katz A, Tanay A, Linhart C, Steinfeld I, Sharan R, Shiloh Y, Elkon R: EXPANDER: an integrative suite for microarray data analysis. BMC Bioinformatics 2005, 6(232):

32. Hohmann $\mathrm{S}$ : Osmotic stress signaling and osmoadaptation in yeasts. Microbiol Mol Biol Rev 2002, 66(2):300-72.

33. O'Rourke SM, Herskowitz I: The Hog I MAPK prevents cross talk between the HOG and pheromone response MAPK pathways in Saccharomyces cerevisiae. Genes Dev 1998, I 2(1 8):2874-2886.

34. Chen $\mathrm{H}$, Xiong L: Pyridoxine is required for post-embryonic root development and tolerance to osmotic and oxidative stresses. Plant Journal 2005, 44(3):396-408.

35. Elkon R, Linhart C, Sharan R, Shamir R, Shiloh Y: Genome-Wide In Silico Identification of Transcriptional Regulators Controlling the Cell Cycle in Human Cells. Genome Research 2003, I3(5):773-780.

36. Olson KA, Nelson C, Tai G, Hung W, Yong C, Astell C, Sadowski I: Two regulators of Stel $2 p$ inhibit pheromone-responsive transcription by separate mechanisms. Mol Cell Biol 2000 20(I 2):4199-209.

37. Martinez-Pastor MT, Marchler G, Schuller C, Marchler-Bauer A, Ruis $H$, Estruch F: The Saccharomyces cerevisiae zinc finger proteins Msn2p and Msn4p are required for transcriptional induction through the stress response element (STRE). $E M B O$ J 1996, I5(9):

38. Whitfield ML, Sherlock G, Saldanha AJ, Murray JI, Ball CA, Alexander KE, Matese JC, Perou CM, Hurt MM, Brown PO, Botstein D: Identification of Genes Periodically Expressed in the Human Cell Cycle and Their Expression in Tumors. Molecular Biology of the Cell 2002, I3:1977-2000.

39. Gavin AC, Aloy P, Grandi P, Krause R, Boesche M, Marzioch M, Rau $C$, Jensen LJ, Bastuck $S$, Dumpelfeld B, Edelmann A, Heurtier MA, Hoffman V, Hoefert C, Klein K, Hudak M, Michon AM, Schelder M, Schirle M, Remor M, Rudi T, Hooper S, Bauer A, Bouwmeester T, Casari G, Drewes G, Neubauer G, Rick JM, Kuster B, Bork P, Russell RB, Superti-Furga G: Proteome survey reveals modularity of the yeast cell machinery. Nature 2006, 440(7084):63I-6.

40. Krogan NJ, Cagney G, Yu H, Zhong G, Guo X, Ignatchenko A, Li J, Pu $S$, Datta N, Tikuisis AP, Punna T, Peregrin-Alvarez JM, Shales M, Zhang X, Davey M, Robinson MD, Paccanaro A, Bray JE, Sheung A, Beattie B, Richards DP, Canadien V, Lalev A, Mena F, Wong P, Starostine A, Canete MM, Vlasblom J, Wu S, Orsi C, Collins SR, Chandran S, Haw R, Rilstone Jj, Gandi K, Thompson NJ, Musso G, St Onge P, Ghanny S, Lam MH, Butland G, Altaf-UI AM, Kanaya S, Shilatifard A O'Shea E, Weissman JS, Ingles CJ, Hughes TR, Parkinson J, Gerstein M, Wodak SJ, Emili A, Greenblatt JF: Global landscape of protein complexes in the yeast Saccharomyces cerevisiae. Nature 2006, 440(7084):637-643.

4I. Ito T, Chiba T, Yoshida M: Exploring the protein interactome using comprehensive two-hybrid projects. Trends Biotechnol 200I, 19:S23-S27.

42. Ho Y, Gruhler A, Heilbut A, Bader GD, Moore L, Adams SL, Millar A, Taylor P, Bennett K, Boutilier K, Yang L, Wolting C, Donaldson I, Schandorff S, Shewnarane J, Vo M, Taggart J, Goudreault M, Muskat B, Alfarano C, Dewar D, Lin Z, Michalickova K, Willems AR, Sassi $H$, Nielsen PA, Rasmussen KJ, Andersen JR, Johansen LE, Hansen LH, Jespersen $H$, Podtelejnikov A, Nielsen E, Crawford J, Poulsen V, Sorensen BD, Matthiesen J, Hendrickson RG, Gleeson F, Pawson T, Moran MF, Durocher D, Mann M, Hogue CW, Figeys D, Tyers M: Systematic identification of protein complexes in Saccharomyces cerevisiae by mass spectrometry. Nature 2002, 4I5: $180-3$

43. Uetz P, Giot L, Cagney G, Mansfield TA, Judson R, Knight JR, Lockshon D, Narayan V, Srinivasan M, Pochart P, Qureshi-Emili A, Li Y, Godwin B, Conover D, Kalbfleisch T, Vijayadamodar G, Yang M, Johnston M, Fields S, Rothberg JM: A comprehensive analysis of pro- tein-protein interactions in Saccharomyces cerevisiae. Nature 2000, 403(6770):623-7.

44. Sharan R, Ideker T, Kelley B, Shamir R, Karp R: Identification of Protein Complexes by Comparative Analysis of Yeast and Bacterial Protein Interaction Data. Journal of Computational Biology 2005, I 2:835-846.

45. Sokal RR, Rohlf F): Biometry: The Principles and Practice of Statistics in Biological Research W.H. Freeman and company; 1995.

46. Charikar M: Greedy Approximation Algorithms for Finding Dense Components in a Graph. Lecture Notes in Computer Science 2000, 1 $913: 84-95$.

47. Holm J, de Lichtenberg K, Thorup M: Poly-logarithmic deterministic fully-dynamic algorithms for connectivity, minimum spanning tree, 2-edge, and biconnectivity. In ProC STOC ' 98 New York, NY, USA: ACM Press; 1998:79-89.

48. Even S: Graph Algorithms Potomac, Maryland: Computer Science Press; 1979.

49. Kashtan N, Itzkovitz S, Milo R, Alon U: Efficient sampling algorithm for estimating subgraph concentrations and detecting network motifs. Bioinformatics 2004, 20(I I): 1746-58.

50. MATISSE web page [http://www.cs.tau.ac.il/ rshamir/matisse/]

51. Everitt B: Cluster analysis third edition. London: Edward Arnold; 1993.

52. McLachlan G], Krishnan T: The EM Algorithm and Extensions John Wiley and Sons, inc; 1997.
Publish with Biomed Central and every scientist can read your work free of charge

"BioMed Central will be the most significant development for disseminating the results of biomedical research in our lifetime. "

Sir Paul Nurse, Cancer Research UK

Your research papers will be:

- available free of charge to the entire biomedical community

- peer reviewed and published immediately upon acceptance

- cited in PubMed and archived on PubMed Central

- yours - you keep the copyright
BioMedcentral 